\title{
A CAUSALITY-BASED CONCURRENT ENGINEERING TOOL TO SUPPORT CUBESAT CONCEPTUAL DESIGN
}

\author{
Stephen Peters ${ }^{1 *}$, Clément Fortin ${ }^{2}$, Grant McSorley ${ }^{1}$ \\ ${ }^{1}$ Faculty of Sustainable Design Engineering, University of Prince Edward Island, Charlottetown, Canada \\ ${ }^{2}$ Skolkovo Institute of Science and Technology, Moscow, Russia \\ *sepeters@upei.ca
}

\begin{abstract}
Modern aerospace conceptual design processes often involve large numbers of personnel from a wide variety of disciplines working on a complex electro-mechanical system. An ongoing challenge is integrating said personnel and their system-related information within a stringent systems engineering environment for the development of a technically complex product. Specific to conceptual design, there is also an intensive iteration process combined with a relative lack of product definition. This leads to problems when using traditional product lifecycle management tools, which rely heavily on the geometry-focused product structure. The objective of this research is to better support conceptual design of CubeSats by developing a software tool to address these issues. Our efforts have been focused on producing a new concurrent engineering tool specifically tailored for the conceptual design phase

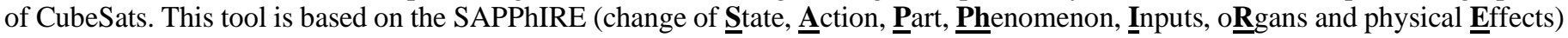
model of causality. It was originally developed to be a flexible representation of both natural and artificial entities to stimulate innovation via analogy, enabling development of novel ideas during conceptual design. More specifically, the use of function, behaviour and structure constructs found in the SAPPhIRE model for illustrating causality shifts reliance from the product structure towards the more consistent system states and resultant behaviour. Despite its flexibility, the model was not originally developed as a knowledge or information management tool for systems development, and so modifications were required to accommodate typical analysis and data files found in conceptual design. To assess its feasibility as a data framework, preliminary work has involved creating high-level SAPPhIRE models consisting of pre-existing files containing conceptual design information related to each subsystem. These representations were used as blueprints for the construction of the design support tool which has been used to further evaluate the capability of the SAPPhIRE model to support conceptual design. This JavaFX software package is based on the preliminary models. Major features include a consolidated location for all engineering information, a dynamic graphical user interface, and a consistent inter-subsystem software architecture to promote integration. During the evaluation period, participants were asked to perform several practical tasks while navigating the structure, Performance and feedback were recorded regarding the software's ability to convey causality among design domains, organize analytical data logically, and its potential to facilitate typical design activities. This presentation will discuss the results and relevant conclusions on the SAPPhIRE model as a data framework to support the conceptual design of CubeSats.
\end{abstract}

